\title{
Gluconeogenesis Inhibition
}

National Cancer Institute

\section{Source}

National Cancer Institute. Gluconeogenesis Inhibition. NCI Thesaurus. Code C40633.

Gluconeogenesis Inhibition involves interference with, or restraint of, the biosynthesis of glucose from 3-carbon non-carbohydrate precursors, including amino acids. 